\title{
Letter to the Editor: Discussion on the Paper "State-of-the-Art of Research on Seismic Pounding Between Buildings with Aligned Slabs"
}

\author{
Robert Jankowski ${ }^{1}$ (D)
}

Received: 29 November 2017 / Accepted: 11 January 2018/ Published online: 19 January 2018

(c) The Author(s) 2018. This article is an open access publication

The paper "State-of-the-art of research on seismic pounding between buildings with aligned slabs" (by Kharazian and López-Almansa) has recently been published in the journal of Archives of Computational Methods in Engineering (see [1]). It can be considered as a kind of continuation of previous 'state-of-the-art' publications, including two papers [2, 3] and a book [4]. Unfortunately, the paper contains a number of misleading or wrong statements as well as mistakes. These errors are discussed and/or corrected below in the order they appear in paper [1].

It is a kind of misunderstanding to consider the classical theory of impact (stereomechanics) as a case of 'concentrated masses connected by a spring with infinitive stiffness', as described in [1]. Actually, the classical theory of impact is based on the laws of conservation of energy and momentum and does not consider stresses and deformations in the colliding elements during impact. It is focused only on determination of velocities of colliding elements after collision without tracing structural response during impact (see [5] for details).

Equation (6) is wrong and its correct form is (see [6]):

$$
\begin{array}{ll}
F=k\left(x_{1}-x_{2}-d\right)+c\left(\dot{x}_{1}-\dot{x}_{2}\right) & \text { if } x_{1}-x_{2}>d \\
F=0 & \text { if } x_{1}-x_{2} \leq d
\end{array}
$$

Equation (9) and two equations at the top of page 7 were proposed in [7]. Equation (9) had originally slightly different form, i.e. (compare [7]):

$$
\begin{array}{ll}
F=k\left(x_{1}-x_{2}-d\right)+c\left(\dot{x}_{1}-\dot{x}_{2}\right) & \text { if } \dot{x}_{1}-\dot{x}_{2}>0 \\
F=k\left(x_{1}-x_{2}-d\right) & \text { if } \dot{x}_{1}-\dot{x}_{2} \leq 0
\end{array}
$$

Equation (10) is wrong and its correct form is (see [8]):

Robert Jankowski

jankowr@pg.edu.pl

1 Faculty of Civil and Environmental Engineering, Gdańsk University of Technology, ul. Narutowicza 11/12, 80-233 Gdańsk, Poland

$$
\begin{array}{ll}
F=\bar{\beta}\left(x_{1}-x_{2}-d\right)^{3 / 2}+\bar{c}\left(\dot{x}_{1}-\dot{x}_{2}\right) & \text { if } \dot{x}_{1}-\dot{x}_{2}>0 \\
F=\bar{\beta}\left(x_{1}-x_{2}-d\right)^{3 / 2} & \text { if } \dot{x}_{1}-\dot{x}_{2} \leq 0
\end{array}
$$

where $\bar{\beta}\left[\mathrm{N} / \mathrm{m}^{3 / 2}\right]$ is the impact stiffness parameter of the non-linear viscoelastic model [instead of $k[\mathrm{~N} / \mathrm{m}]$ which is stiffness of the Kelvin-Voigt model defined in Eq. (6)] and $\bar{c}$ is the non-linear impact element's damping [which is also different than damping $c$ used in the Kelvin-Voigt model defined in Eq. (6)].

That is not true that the objective of the non-linear viscoelastic model, proposed in [8], is 'to eliminate the jumps that appear in the modified Kelvin-Voigt model at the beginning and the end of the contact'. Actually, the aim of the non-linear viscoelastic model is to simulate pounding force during impact more precisely comparing to the normal (not modified) Kelvin-Voigt model (see [8] for details). That aim is obtained by applying a non-linear spring following the Hertz law of contact which more accurately simulates the relation between force and deformation. Additionally, a non-linear damper is activated only during the approach period of collision in order to simulate the process of energy dissipation which takes place mainly during that period [5].

Equation (11) is wrong and its correct form is (see [9]):

$$
\begin{array}{ll}
F=\left(x_{1}-x_{2}-d\right)^{3 / 2}\left[k_{h}+\zeta\left(\dot{x}_{1}-\dot{x}_{2}\right)\right] & \text { if } x_{1}-x_{2}>d \\
F=0 & \text { if } x_{1}-x_{2} \leq d
\end{array}
$$

where $k_{h}\left[\mathrm{~N} / \mathrm{m}^{3 / 2}\right]$ is the impact stiffness parameter of the Hertzdamp model [instead of $k[\mathrm{~N} / \mathrm{m}]$ which is stiffness of the Kelvin-Voigt model defined in Eq. (6)] and:

$\zeta=\frac{3 k_{h}\left(1-r^{2}\right)}{4\left(v_{1}-v_{2}\right)}$

where $v_{1}-v_{2}$ is the relative approaching velocity (see [9] for details).

It is difficult to agree with the sentence that 'the models based on the Hertz contact law are not adequate, since that law was derived for elastic contact between balls', as 
described in [1]. On the contrary to the above statement, the results of the analyses (see, for example [8]) indicate that the non-linear Hertz elastic model can be very effective in modelling earthquake-induced structural pounding when the loss of energy during collisions is not so significant. Moreover, in such cases (due to its non-linear relation between force and deformation) it can be even more accurate than the linear Kelvin-Voigt model (see [8]).

It is also difficult to agree with one of the conclusions of the paper [1] that 'in pounding simulation, it is basically suggested to utilize the normal Kelvin-Voigt model'. Well, the truth is that the normal Kelvin-Voigt model is a simple and quite accurate one. It can also be easily implemented in the computer programs. It should be underlined, however, that the linear relation between force and deformation, assumed in the model, is not fully consistent with the reality and, moreover, a model produces a negative impact force just before separation which does not have a physical explanation [5]. In order to eliminate the above shortcomings, more precise models, including non-linear viscoelastic model and Hertzdamp model (both described also in [1]), have been proposed. These models allow us to obtain more accurate response time histories of colliding structures under earthquake excitation (see, for example, the results of comparisons shown in [8]), although they require more efforts to be implemented in the computer programs.

\section{Compliance with Ethical Standards}

Conflict of interest The author declares that he has no conflict of interest.
Open Access This article is distributed under the terms of the Creative Commons Attribution 4.0 International License (http://creative commons.org/licenses/by/4.0/), which permits unrestricted use, distribution, and reproduction in any medium, provided you give appropriate credit to the original author(s) and the source, provide a link to the Creative Commons license, and indicate if changes were made.

\section{References}

1. Kharazian A, López-Almansa F (2017) State-of-the-art of research on seismic pounding between buildings with aligned slabs. Arch Computat Methods Eng. https://doi.org/10.1007/s11831-017-92423

2. Anagnostopoulos SA (1995) Earthquake induced pounding: State of the art. In: Proceedings of the 10th European conference on earthquake engineering on 28.08-2.09.1994, Vienna, Austria, pp 897-905

3. Cole GL, Dhakal RP, Carr AJ, Bull DK (2010) Building pounding state of the art: identifying structures vulnerable to pounding damage. In: 2010 NZSEE conference on 26-28.03.2010, Wellington, New Zealand

4. Jankowski R, Mahmoud S (2015) Earthquake-induced structural pounding. Springer, Switzerland. https://doi.org/10.1007/978-3319-16324-6

5. Goldsmith W (1960) Impact: the theory and physical behaviour of colliding solids. Edward Arnold, London

6. Anagnostopoulos SA (1988) Pounding of buildings in series during earthquakes. Earthq Eng Struct Dyn 16:443-456

7. Jankowski R, Mahmoud S (2011) Modified linear viscoelastic model of earthquake-induced structural pounding. Iran J Sci Technol 35(C1):51-62

8. Jankowski R (2005) Non-linear viscoelastic modelling of earthquake-induced structural pounding. Earthq Eng Struct Dyn 34(6):595-611

9. Muthukumar S, DesRoches R (2006) A Hertz contact model with non-linear damping for pounding simulation. Earthq Eng Struct Dyn 35(7):811-828 